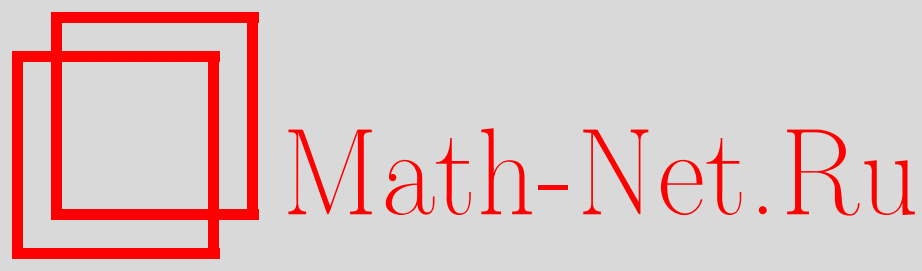

А. М. Поволоцкий, В. Б. Приезжев, Многоточечные пространственно-временные переходы в простом полностью асимметричном процессе с исключающим взаимодействием, ТМФ, 2011, том 169, номер 1, 167-175

DOI: https://doi.org/10.4213/tmf6718

Использование Общероссийского математического портала Math-Net.Ru подразумевает, что вы прочитали и согласны с пользовательским соглашением http://www.mathnet.ru/rus/agreement

Параметры загрузки:

IP: 3.80 .181 .102

26 апреля 2023 г., 15:58:57

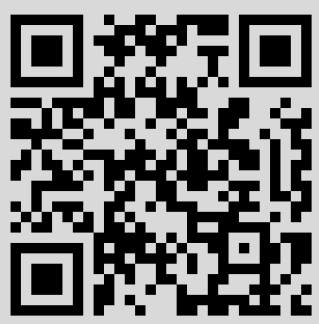




\title{
ПРОСТРАНСТВЕННО-ВРЕМЕННЫЕ ПЕРЕХОДЫ В ПРОСТОМ ПОЛНОСТЬЮ АСИММЕТРИЧНОМ ПРОЦЕССЕ С ИСКЛЮЧАЮЩИМ ВЗАИМОДЕЙСТВИЕМ
}

\begin{abstract}
Изучаются корреляционные функции простого полностью асимметричного процесса с исключающим взаимодействием в дискретном времени с обратной последовательной динамикой. Доказывается детерминантная формула для обобщенной функции Грина, которая описывает переходы между положениями частиц в заданные моменты времени. В качестве примера вычисляется корреляционная функция токов, т. е. совместное распределение вероятностей времен, необходимых каждой частице, чтобы пройти данное расстояние. Асимптотический анализ показывает, что флуктуации токов сходятся к процессу Airy 2 .
\end{abstract}

Ключевые слова: простой полностью асимметричный процесс с исключающим взаимодействием, анзац Бете, детерминантный точечный процесс, класс универсальности Кардара-Паризи-Жанга.

\section{1. ВВЕДЕНИЕ}

В последнее время простой полностью асимметричный процесс с исключающим взаимодействием (ППАП) стал объектом интенсивного изучения [1]-[4]. В частности, для ППАП на бесконечной решетке было получено несколько точных результатов для переходных вероятностей, зависящих от времени, и среди них детерминантные формулы для функций Грина. Эти функции, являющиеся переходными вероятностями между конфигурациями частиц в различные моменты времени, были впервые получены в работе [5] для ППАП в непрерывном времени, а затем обобщены на случаи дискретной динамики [6]-[8] и геометрии кольца [9], [10].

Следующим достижением стало вычисление корреляционных функций, т. е. переходных вероятностей для нескольких выделенных частиц при условии, что по координатам остальных частиц произведено интегрирование. Первый результат состоял

* Объединенный институт ядерных исследований, Дубна, Московская обл., Россия. E-mail: alexander.povolotsky@gmail.com, priezzvb@theor.jinr.ru 
в нахождении распределения координаты $N$-й частицы в произвольный момент времени для ППАП с параллельной динамикой и ступенчатыми начальными условиями [11]. Этот результат был обобщен затем на обратную последовательную динамику [7] и распространен на однородные начальные условия [12]. Метод нахождения многочастичных распределений был предложен в работе [13]. Затем было проведено подробное изучение распределения координат выделенных частиц в фиксированный момент времени [14]-[16], координаты одной выделенной частицы в различные моменты времени [17], распределения координат частиц на пространственноподобных траекториях [18], [19]. Целью настоящей работы является расширение области, доступной для вычисления многочастичных распределений. Мы рассмотрим ППАП с обратной последовательной динамикой, ступенчатыми начальными условиями и найдем объединенное распределение времен, необходимых каждой из выделенных частиц, чтобы пройти заданное расстояние.

Особый интерес представляет скейлинговое поведение полученных распределений. Из известных ранее результатов следует, что скейлинговое поведение характеризуется классом универсальности Кардара-Паризи-Жанга (КПЖ) [20], и при этом предельные распределения сходятся к соответствующим распределениям универсальных случайных процессов таких, как Airy 1 [13], Airy 2 [21] и т. д. в зависимости от глобальной формы профиля начальной плотности. Асимптотический анализ показывает, что в нашем случае многочастичные распределения времен сходятся к процессу Airy 2 . Это согласуется с утверждением о тождественности корреляций вдоль различных пространственно-временнь́х направлений, которое основано на явлении медленной декорреляции, обнаруженной в работе [22].

Ниже мы дадим краткое изложение результатов, приводя лишь определения и формулировки теорем без доказательств. Детали доказательств можно найти в работе [23].

\section{2. ППАП В ДИСКРЕТНОМ ВРЕМЕНИ}

Рассмотрим $N$ частиц на целочисленной одномерной решетке. Конфигурация системы $\boldsymbol{x}$ принимает значения на множестве $\left\{\left(x_{1} \cup \cdots \cup x_{N}\right) \in \mathbb{Z}\right\}$, так что $N$ строго возрастающих целых чисел $x_{1}>x_{2}>\cdots>x_{N}$ обозначают упорядоченные координаты частиц. Строго возрастающий порядок отвечает условию исключения: две частицы не могут одновременно находиться в одном узле.

Марковская динамика этого процесса за один дискретный шаг времени может быть определена следующим образом. Начиная с крайней правой частицы с координатой $x_{1}<\infty$, мы допускаем прыжок каждой частицы в соседний правый узел с вероятностью $p$ при условии, что этот узел свободен. Если этот узел занят, то вероятность частицы остаться на месте равна 1. Следовательно, ППАП - случайный процесс, заданный как последовательность конфигураций $\boldsymbol{x}^{0}, \boldsymbol{x}^{1}, \ldots, \boldsymbol{x}^{t}$. Мы называем такую последовательность траекторией системы за время $t$. Каждая траектория реализуется с вероятностью

$$
P\left(\boldsymbol{x}^{0}, \ldots, \boldsymbol{x}^{t}\right)=P_{1}\left(\boldsymbol{x}^{t} \mid \boldsymbol{x}^{t-1}\right) \ldots P_{1}\left(\boldsymbol{x}^{2} \mid \boldsymbol{x}^{1}\right) P_{1}\left(\boldsymbol{x}^{1} \mid \boldsymbol{x}^{0}\right) P_{0}\left(\boldsymbol{x}^{0}\right),
$$

где $P_{0}(\boldsymbol{x})$ - вероятность начальной конфигурации $\boldsymbol{x}$. Вероятность одного шага $P_{1}(\boldsymbol{x} \mid \boldsymbol{y})$ из конфигурации $\boldsymbol{y}$ в $\boldsymbol{x}$ имеет вид

$$
P_{1}(\boldsymbol{x} \mid \boldsymbol{y})=\prod_{i=1}^{N} \theta\left(x_{i}-y_{i}, x_{i-1}-y_{i}\right),
$$


где $\theta(k, l)=\left(q+p \delta_{l, 1}\right) \delta_{k, 0}+p \delta_{k, 1}$, и мы формально считаем, что $x_{0}=\infty$. Параметр $p$, вероятность прыжка, изменяется в пределах $0<p<1$, и мы определяем $q=1-p$. Заданные таким образом переходные вероятности соответствуют обратной последовательной динамике в дискретном времени, когда сначала перемещается первая частица, за ней вторая и т. д. Поскольку движение частиц полностью асимметрично, первые $N$ частиц "не чувствуют" присутствия остальных частиц, находящихся слева. Следовательно, рассматривая динамику первых $N$ частиц, мы можем без ограничения общности изучать динамику в точности $N$ частиц с самого начала. Тогда для любого фиксированного $t$ число конфигураций, а также число траекторий, реализующихся с ненулевой вероятностью, остается конечным.

\section{3. ФУНКЦИИ ГРИНА}

Рассмотрим вероятность системы находиться в состоянии $\boldsymbol{x}$ после $t$ шагов, $t \geqslant 0$ :

$$
G_{t}(\boldsymbol{x} \mid \boldsymbol{y})=\sum_{\left\{\boldsymbol{x}^{0}, \boldsymbol{x}^{1}, \ldots, \boldsymbol{x}^{t-1}\right\}} P\left(\boldsymbol{x}^{0}, \ldots, \boldsymbol{x}^{t}\right)
$$

при данном $P_{0}\left(\boldsymbol{x}^{0}\right)=\delta_{\boldsymbol{y}, \boldsymbol{x}^{0}}$. Суммирование производится по всем траекториям, ведущим в заданную конечную конфигурацию; $\boldsymbol{x}^{t} \equiv \boldsymbol{x}$. Явная форма $G_{t}(\boldsymbol{x}, \boldsymbol{y})$ для ППАП особенно проста и дается детерминантом матрицы размером $N \times N$ [5], которая для ППАП с обратной последовательной динамикой имеет следующий вид [6], [7].

ПреДЛОЖЕНИЕ 3.1. Функиия Грина имеет вид детерминанта:

$$
G_{t}(\boldsymbol{x} \mid \boldsymbol{y})=\operatorname{det}\left[F_{j-i}\left(x_{i}-y_{j}, t\right)\right]_{i, j=1, \ldots, N},
$$

¿əe

$$
F_{n}(x, t)= \begin{cases}\frac{1}{2 \pi i} \oint_{\Gamma_{0}} \frac{d w}{w}\left(q+\frac{p}{w}\right)^{t}(1-w)^{-n} w^{x}, & t \geqslant 0 \\ 0, & t<0\end{cases}
$$

и контур интегрирования $\Gamma_{0}$ охватывает начало координат, оставляя точку $w=1$ вне контура.

В настоящей статье мы собираемся рассмотреть ППАП на множестве пространственно-временны́х точек, связанном с различными моментами времени. Для этого мы введем обобщенную функцию Грина (ОФГ) [24], которая может быть описана на языке непересекающихся решеточных путей. Рассмотрим двумерную целочисленную решетку $L=\mathbb{Z}^{2}$. Каждая точка этой решетки определяется парой целых чисел $(x, t)$, где $x$ соответствует положению частицы, а $t$ - дискретному времени. Фиксируем две точки $\left(x^{0}, t^{0}\right) \in L$ и $(x, t) \in L$ такие, что $t>t_{0}$. Направленный решеточный путь длины $\left(t-t_{0}\right)$ из начальной точки $\left(x^{0}, t^{0}\right)$ в конечную точку $(x, t)-$ подмножество $L$ вида

$$
\Pi_{(x, t)}^{\left(x^{0}, t^{0}\right)}=\bigcup_{i=0}^{t-t^{0}}\left\{\left(x^{i}, t^{i}\right)\right\}
$$

где $x^{t-t^{0}} \equiv x, t^{i}=t^{0}+i$ и $x^{i} \in \mathbb{Z}$ может принимать любые значения для $i=$ $1, \ldots, t-t^{0}-1$. 
Каждому пути мы приписываем вес $W\left(\Pi_{(x, t)}^{\left(x^{0}, t^{0}\right)}\right)$, определенный как произведение весов элементарных двухточечных путей

$$
W\left[\Pi_{(x, t)}^{\left(x^{0}, t^{0}\right)}\right]=\prod_{i=0}^{t-t^{0}-1} W\left[\left\{\left(x^{i}, t^{i}\right),\left(x^{i+1}, t^{i+1}\right)\right\}\right] .
$$

Решеточный путь представляет стохастическую траекторию частицы. Соответственно, множество ненулевых элементарных весов определено условием разрешенных прыжков частиц. Например, для одномерного направленного случайного блуждания Бернулли, которое является частным случаем рассматриваемой динамики, ненулевые элементарные веса определены как $W(\{(x, t),(x, t+1)\})=q$ и $W(\{(x, t)$, $(x+1, t+1)\})=p$, где $(x, t) \in \mathbb{Z}^{2}$.

Для обобщения результатов, полученных для $N$-частичной функции Грина, мы должны рассмотреть $N$ взаимодействующих решеточных путей. Заметим, что вид функции Грина, соединяющей две конфигурации частиц в разные моменты времени, определяется тем фактом, что частицы не сталкиваются и остаются упорядоченными в течение всей эволюции. Это остается справедливым для пространственно-временнь́х траекторий, т. е. для соответствующих решеточных путей. Будучи пронумерованными в начальный момент времени, точки, лежащие на различных путях, сохраняют тот же порядок в любой последующий момент времени. Мы хотим построить обобщение функции Грина таким образом, чтобы начальные и конечные положения частиц соответствовали различным моментам времени. Для того чтобы такое определение было полезным на практике, должно выполняться условие упорядоченности путей. Это оказывается возможным, если ограничить область начальных и конечных конфигураций условием их допустимости.

Множество $N$ упорядоченных точек $L(\boldsymbol{x}, \boldsymbol{t})=\left(\left(x_{1}, t_{1}\right), \ldots,\left(x_{N}, t_{N}\right)\right)$ называется допустимым, если условия $x_{1}>x_{2}>\cdots>x_{N}$ и $t_{1} \leqslant t_{2} \leqslant \cdots \leqslant t_{N}$ выполняются одновременно. Тогда для двух допустимых $N$-точечных конфигураций $\left(\boldsymbol{x}^{\mathbf{0}}, \boldsymbol{t}^{\mathbf{0}}\right)$ и $(\boldsymbol{x}, \boldsymbol{t})$ таких, что $t_{i}>t_{i}^{0}$ при $i=1, \ldots, N$, мы определяем $N$-путь как подмножество $L \times\{1, \ldots, N\}$ вида

$$
\boldsymbol{\Pi}_{(\boldsymbol{x}, \boldsymbol{t})}^{\left(\boldsymbol{x}_{0}, \boldsymbol{t}_{0}\right)}=\left\{\left(\Pi_{1}\right)_{\left(x_{1}, t_{1}\right)}^{\left(x_{1}^{0}, t_{1}^{0}\right)}, \ldots,\left(\Pi_{N}\right)_{\left(x_{N}, t_{N}\right)}^{\left(x_{N}^{0}, t_{N}^{0}\right)}\right\}
$$

Число $T=t_{N}-t_{1}^{0}$ будем считать длиной $N$-пути.

Взаимодействие в ППАП приводит к тому, что статистический вес $W[\boldsymbol{\Pi}] N$-пути не является, вообще говоря, произведением весов $N$ единичных путей. А именно, он имеет вид произведения членов, зависящих от расположения двух смежных путей,

$$
W[\mathbf{\Pi}]=W\left[\Pi_{1}\right] \prod_{i=1}^{N-1} W\left[\Pi_{i+1} \mid \Pi_{i}\right]
$$

Вес $W\left[\Pi_{i+1} \mid \Pi_{i}\right]$ есть произведение двухточечных членов для второго пути $\Pi_{i+1}$ :

$$
W\left[\Pi_{i+1} \mid \Pi_{i}\right]=\prod_{j=0}^{t_{i+1}-t_{i+1}^{0}-1} W\left[\left\{\left(x_{i+1}^{j}, t_{i+1}^{j}\right),\left(x_{i+1}^{j+1}, t_{i+1}^{j+1}\right)\right\} \mid \Pi_{i}\right],
$$


при условии, что конфигурация первого пути $\Pi_{i}$. Все ненулевые двухточечные члены определены как

$$
\begin{gathered}
W[\{(x, t),(x+1, t+1)\} \mid \Pi]= \begin{cases}p, & (x+1, t+1) \notin \Pi, \\
0, & (x+1, t+1) \in \Pi,\end{cases} \\
W[\{(x, t),(x, t+1)\} \mid \Pi]= \begin{cases}q, & (x+1, t+1) \notin \Pi, \\
1, & (x+1, t+1) \in \Pi .\end{cases}
\end{gathered}
$$

Теперь мы можем определить ОФГ.

Для двух допустимых $N$-точечных конфигураций $(\boldsymbol{x}, \boldsymbol{t})$ и $\left(\boldsymbol{x}_{0}, \boldsymbol{t}_{0}\right)$ таких, что $\boldsymbol{t}_{i}>$ $t_{i}^{0}$, ОФГ из $\left(\boldsymbol{x}_{0}, \boldsymbol{t}_{0}\right)$ в $(\boldsymbol{x}, \boldsymbol{t})$ есть

$$
G\left(\left(\boldsymbol{x}, \boldsymbol{t}\left(\boldsymbol{x}_{0}, \boldsymbol{t}_{0}\right)\right)=\sum_{\left\{\boldsymbol{\Pi}_{(\boldsymbol{x}, \boldsymbol{t})}^{\left(\boldsymbol{x}^{0}, \boldsymbol{t}^{0}\right)}\right\}} W\left[\boldsymbol{\Pi}_{(\boldsymbol{x}, \boldsymbol{t})}^{\left(\boldsymbol{x}^{0}, \boldsymbol{t}^{0}\right)}\right],\right.
$$

где суммирование проводится по всем возможным $N$-путям между $\left(\boldsymbol{x}^{\mathbf{0}}, \boldsymbol{t}^{\mathbf{0}}\right)$ и $(\boldsymbol{x}, \boldsymbol{t})$.

Очевидно, что функция Грина $G_{t}\left(\boldsymbol{x} \mid \boldsymbol{x}_{\mathbf{0}}\right)$, определенная в начале настоящего раздела, является частным случаем ОФГ с $t_{1}^{0}=\cdots=t_{N}^{0} \equiv t^{0}$ и $t_{1}=\cdots=t_{N}=t+t^{0}$. Разумеется, она не зависит от $t^{0}$ из-за инвариантности по отношению к сдвигу времени. Существенно то, что детерминантная форма (4) для $G_{t}\left(\boldsymbol{x} \mid \boldsymbol{x}_{\mathbf{0}}\right)$ обобщается на ОФГ (см. также работу [24]).

ПрЕДЛОЖЕНИЕ 3.2. Пусть данъ $N$-точечные конфигурачии $(\boldsymbol{x}, \boldsymbol{t})$ u $\left(\boldsymbol{x}_{0}, \boldsymbol{t}_{0}\right)$ maкие, что $t_{i}>t_{i}^{0}$ для $i=1, \ldots, N$. Тогда ОФГ может быть выражена в детерминантном виде:

$$
G\left((\boldsymbol{x}, \boldsymbol{t}) \mid\left(\boldsymbol{x}_{0}, \boldsymbol{t}_{0}\right)\right)=\operatorname{det}\left[F_{j-i}\left(x_{i}-x_{j}^{0}, t_{i}-t_{j}^{0}\right)\right]_{i, j=1, \ldots, N},
$$

где $F_{n}(x, t)$ определено формулой (5).

\section{4. ЗНАКОПЕРЕМЕННЫЙ ДЕТЕРМИНАНТНЫЙ ПРОЦЕСС}

Рассмотрим $N$ частиц, начиная с момента времени $t=0$, когда они находятся в разделенных единичными интервалами узлах, $x_{i}^{0}=1-i$. Наша задача - найти совместное распределение

$$
\mathbf{P}=\operatorname{Prob}\left(\left\{t_{n_{1}} \leqslant a_{1}\right\} \cap\left\{t_{n_{2}} \leqslant a_{2}\right\} \cap \cdots \cap\left\{t_{n_{m}} \leqslant a_{m}\right\}\right)
$$

времен $t_{n_{1}}, \ldots, t_{n_{m}}$, когда $m \leqslant N$ частиц с номерами $n_{1}<n_{2}<\cdots<n_{m}$ выходят из узлов $x_{n_{1}}, \ldots, x_{n_{m}}$, выбранных из множества $x_{i}=x+N-i, i=1, \ldots, N$, в предположении $t_{n_{1}} \leqslant t_{n_{2}} \leqslant \cdots \leqslant t_{n_{m}}$. Для этого рассмотрим множество $N$-путей, состоящих из $N$ путей, исходящих из точек $(x-i+N, 0)$ и делающих последний шаг из точек $\left(x-i+N, t_{i}\right)$ в $\left(x-i+N+1, t_{i}+1\right)$, для всех $i=1, \ldots, N$ соответственно. Вероятность некоторого набора $t_{1}, \ldots, t_{N}$ дается ОФГ $G\left((\boldsymbol{x}, \boldsymbol{t}) \mid\left(\boldsymbol{x}^{\mathbf{0}}, \boldsymbol{t}^{\mathbf{0}}\right)\right)$, умноженной на вероятность последнего шага $p^{N}$. Искомая величина (15) есть частная вероятность такой $N$-частичной вероятности. Ее вывод значительно упростится, если мы заметим, что ОФГ сама может быть представлена как частная вероятность вспомогательного детерминантного точечного процесса [25]. 
Рассмотрим знакопеременный точечный процесс на подмножествах вида

$$
\mathcal{T}=\bigcup_{1 \leqslant n \leqslant N}\left\{\tau_{n}^{n}<\tau_{n-1}^{n}<\cdots<\tau_{1}^{n}\right\} \subset \mathbb{Z}_{\geqslant(x-1)} \times\{1, \ldots, n\}
$$

множества $\mathbb{Z}_{\geqslant x} \times\{1, \ldots, n\}$, определенный как мера

$$
\mathcal{F}(\mathcal{T})=\frac{1}{Z_{N}} \prod_{n=0}^{N-1} \operatorname{det}\left[\phi_{n}\left(\tau_{i}^{n}, \tau_{j}^{n+1}\right)\right]_{i, j=1}^{n+1} \operatorname{det}\left[\Psi_{i}^{N}\left(\tau_{N-i}^{N}\right)\right]_{i, j=0}^{N-1},
$$

где мы ввели функции

$$
\begin{gathered}
\phi_{n}(z, y)= \begin{cases}p, & y \geqslant z, \\
0, & y<z,\end{cases} \\
\Psi_{k}^{N}(t)=(-1)^{k} \widetilde{F}_{-k}(x+N-k-1, t),
\end{gathered}
$$

где

$$
\widetilde{F}_{n}(x, t)=\frac{1}{2 \pi i} \oint_{\Gamma_{0}} \frac{d w}{w}\left(q+\frac{p}{w}\right)^{t}(1-w)^{-n} w^{x} .
$$

Это интегральное представление справедливо для $t \in \mathbb{Z}$, что отличается от определения (5) функции $F_{n}(x, t)$, которая совпадает с $\widetilde{F}_{n}(x, t)$, когда $t \geqslant 0$, и равна нулю при $t<0$.

Кроме того, мы ввели фиктивные переменные $\tau_{n}^{n-1}, 1 \leqslant n \leqslant N$, которые по определению всегда меньше $\tau_{j}^{n}$, так что $\phi_{n}\left(\tau_{n+1}^{n}, \tau_{j}^{n+1}\right) \equiv p$ для $j=1, \ldots, n+1$. Если мы рассмотрим $\tau_{j}^{n}$ как координаты вспомогательных частиц на $n$-м временно́м шаге, то $\tau_{n+1}^{n}$ будет соответствовать частице, входящей в систему из резервуара слева [13].

После того как мы определили меру $\mathcal{M}(\mathcal{T})$, можно дать ОФГ следующую интерпретацию.

ПРЕДЛОЖЕНИЕ 4.1. Пусть

$$
\begin{aligned}
\left(\boldsymbol{x}^{0}, \boldsymbol{t}^{0}\right) & =((0,0), \ldots,(-N+1,0)), \\
(\boldsymbol{x}, \boldsymbol{t}) & =\left(\left(x+N-1, t_{1}\right), \ldots,\left(x, t_{N}\right)\right)
\end{aligned}
$$

nри $t_{1} \leqslant t_{2} \leqslant \cdots \leqslant t_{N} \in \mathbb{Z}_{\geqslant x}$ u $x \geqslant-N+1$. Тогда

$$
p^{N} G\left((\boldsymbol{x}, \mathbf{t}) \mid\left(\boldsymbol{x}^{\mathbf{0}}, \boldsymbol{t}^{\mathbf{0}}\right)\right)=\mathcal{M}\left(\bigcup_{k=1}^{N}\left\{\tau_{1}^{k}=t_{k}\right\}\right) .
$$

Мера $\mathcal{M}(\mathcal{T})$ имеет такую же функциональную форму, как аналогичная мера в работах [13], [14], [25]. В частности, к ней можно применить лемму 3.4 из работы [14]. Отличие состоит только в виде функций $\Psi_{n}^{N}(t)$ и в форме пространства, где живут $\tau_{j}^{i}$, которое в нашем случае $\mathbb{Z}_{\geqslant x}$, а не $\mathbb{Z}$. Эта разница не влияет на применимость леммы, сформулированной в абстрактных терминах, хотя и должна учитываться при получении конечных выражений. В соответствии с леммой многоточечные корреляционные функции $\mathcal{M}(\mathcal{T})$ имеют детерминантную форму. 
ПРЕДЛОЖЕНИЕ 4.2. Корреляционное ядро меръ $\mathcal{M}(17)$ имеет вид

$$
\begin{aligned}
& K\left(n_{1}, \tau_{1} ; n_{2}, \tau_{2}\right)= \\
& =p \oint_{\Gamma_{1}} \frac{d v}{2 \pi i v} \oint_{\Gamma_{0, v}} \frac{d w}{2 \pi i w} \frac{(1-p((w-1) / w))^{\tau_{1}}((w-1) / w)^{n_{1}}(w / v)^{x+N}}{(1-p((v-1) / v))^{\tau_{2}+1}((v-1) / v)^{n_{2}}(w-v)}- \\
& \quad-\mathbb{1}\left(n_{2}>n_{1}\right) \oint_{\Gamma_{1,0}} \frac{p d z}{2 \pi i z^{2}}\left(\frac{z-1}{z}\right)^{n_{1}-n_{2}}\left(1-p \frac{z-1}{z}\right)^{\tau_{1}-\tau_{2}-1} .
\end{aligned}
$$

\section{5. КОРРЕЛЯТОРЫ ТОКОВ}

Результаты, полученные в предыдущих разделах, дают нам все, что необходимо для записи искомой вероятности (15) в виде детерминанта Фредгольма. Для этого сформулируем следующую теорему.

Теорема 5.1. Справедливо выражение

$$
\left.\mathbf{P}=\operatorname{det}\left(\mathbb{1}-\chi_{a} K \chi_{a}\right)\right)_{l^{2}\left(\left\{n_{1}, \ldots, n_{m}\right\} \times \mathbb{Z}_{\geqslant x}\right)},
$$

где $\chi_{a}\left(n_{i}\right)(t)=\mathbb{1}\left(t>a_{i}\right)$.

Нас интересует поведение полученного детерминанта Фредгольма в скейлинговом режиме. Посмотрим на частицу с номером $n$, выходящую из узла $x_{n}$ в момент времени $t_{n}$, и рассмотрим скейлинг

$$
n=\nu L, \quad x_{n}=\chi L, \quad t_{n}=\omega L,
$$

где $L$ - большой параметр. В пределе $L \rightarrow \infty$ случайная переменная $\omega$ становится детерминистски связанной с переменными $\gamma$ и $\nu$.

В соответствии с законом больших чисел для стохастической динамики частиц [26], перемасштабированные переменные удовлетворяют соотношению

$$
\omega=\frac{1}{p}(\sqrt{q \nu}+\sqrt{\chi+\nu})^{2}
$$

которое выполняется, когда $p /(p-1) \leqslant \chi / \omega \leqslant p$. В нашем случае конечные положения частиц фиксированы: $x_{n}=x+N-n$. Следовательно, отношение $\gamma \equiv(x+N) / L=\chi+\nu$ фиксировано, когда $L$ стремится к бесконечности. Введем функцию [7]

$$
\omega(\nu)=\frac{1}{p}(\sqrt{q \nu}+\sqrt{\gamma})^{2},
$$

где $\nu \geqslant 0, \gamma \geqslant 0$, описывающую наиболее вероятное время, когда $n$-я частица выйдет из узла $x_{n}$.

Скейлинговый предел корреляционных функций нужно понимать как корреляции между моментами прыжков частиц с номерами, близкими к некоторой точке детерминистской шкалы, отличающимися на характерном для класса универсальности КПЖ масштабе, где и ожидается нетривиальное скейлинговое поведение. Выберем $\left|n_{i}-n_{j}\right| \sim L^{2 / 3}$, тогда как доминирующий масштаб флуктуаций времени $\delta t_{i} \sim L^{1 / 3}$. Эта шкала определяет область, вносящую основной вклад в детерминант Фредгольма. Если опустить множество необходимых математических 
подробностей, можно охарактеризовать асимптотическое поведение детерминанта Фредгольма, проанализировав корреляционное ядро с помощью метода наискорейшего спуска. Такой анализ показывает, что ядро асимптотически стремится к ядру $\operatorname{Airy}_{2}$.

ЛЕмма. Введем новые переменные $u_{i}, s_{i}, i=1,2$, такие, что

$$
n_{i}=\left[L+u_{i} L^{2 / 3}\right], \quad \tau_{i}=\left[L \omega\left(1+u_{i} L^{-1 / 3}\right)+L^{1 / 3} s_{i}\right]
$$

и остающиеся конечными при $L \rightarrow \infty$, а также $K$-ядро из предложения 4.2. Тогда

$$
\lim _{L \rightarrow \infty} L^{1 / 3} K\left(n_{1}, \tau_{1} ; n_{2}, \tau_{2}\right) \simeq \kappa_{t} K_{\mathrm{Airy}_{2}}\left(\kappa_{h} u_{1}, \kappa_{t} s_{1} ; \kappa_{h} u_{2}, \kappa_{t} s_{2}\right)
$$

где в правой части имеем расширенное ядро Эйри

$$
\begin{aligned}
K_{\mathrm{Airy}_{2}}\left(\xi_{1}, \zeta_{1} ; \xi_{2}, \zeta_{2}\right)= \\
\quad= \begin{cases}\int_{0}^{\infty} d \lambda e^{\lambda\left(\xi_{2}-\xi_{1}\right)} A i\left(\lambda+\zeta_{1}\right) A i\left(\lambda+\zeta_{2}\right), & \xi_{2} \leqslant \xi_{1}, \\
-\int_{-\infty}^{0} d \lambda e^{\lambda\left(\xi_{2}-\xi_{1}\right)} A i\left(\lambda+\zeta_{1}\right) A i\left(\lambda+\zeta_{2}\right), & \xi_{2}>\xi_{1},\end{cases}
\end{aligned}
$$

$a$

$$
\begin{aligned}
\kappa_{h} & =\frac{q^{1 / 6} \gamma^{1 / 3}}{2(1+\sqrt{q \gamma})^{1 / 3}(\sqrt{\gamma}+\sqrt{q})^{1 / 3}}, \\
\kappa_{t} & =\frac{p q^{-1 / 6} \gamma^{1 / 6}}{(1+\sqrt{q \gamma})^{2 / 3}(\sqrt{\gamma}+\sqrt{q})^{2 / 3}}
\end{aligned}
$$

- модельно-зависимые масштабные константы. Символ $\simeq$ означает равенство с точностью до матричного сопряљения, которое не влияет на значения миноров $K$.

Константа $\kappa_{t}$ возникала раньше [7], [11]. Данная лемма вместе с аргументами, обосновывающими предельный переход под знаком детерминанта Фредгольма, приводят к следующей теореме, описывающей универсальное поведение перемасштабированного процесса.

Теорема 5.2. Следующий предел справедлив в смысле конечномерных распределений:

$$
\lim _{L \rightarrow \infty} \frac{t_{L+u L^{2 / 3}}-L \omega\left(1+u L^{-1 / 3}\right)}{L^{1 / 3}}=\kappa_{t} \mathcal{A}_{2}\left(\kappa_{h} u\right)
$$

где $\mathcal{A}_{2}$ - процесс $\mathrm{Airy}_{2}$, характеризуемый многоточечными распределениями:

$$
\operatorname{Prob}\left(\mathcal{A}_{2}\left(u_{1}\right)<s_{1}, \ldots, \mathcal{A}_{2}\left(u_{m}\right)<s_{m}\right)=\operatorname{det}\left(\mathbb{1}-\chi_{s} K_{\mathrm{Airy}_{2}} \chi_{s}\right)_{L^{2}\left(\left\{n_{1}, \ldots, n_{m}\right\} \times \mathbb{R}\right)} .
$$

Благодарности. Работа поддержана грантами РФФИ № 07-02-91561a, 09-0100271а и DFG № 436 RUS 113/909/0-1(R). 


\section{Список литературы}

[1] T.M. Liggett, Stochastic Interacting Systems: Contact, Voter and Exclusion Processes, Fundamental Principles of Mathematical Sciences, 324, Springer, Berlin, 1999.

[2] H. Spohn, Large scale dynamics of interacting particles, Texts and Monographs in Physics, 342, Springer, Berlin, 1991.

[3] B. Derrida, Phys. Rep., 301:1-3 (1998), 65-83.

[4] G. M. Schütz, "Exactly solvable models for many-body systems far from equilibrium", Phase Transitions and Critical Phenomena, 19, eds. C. Domb, J.L. Lebowitz, Academic Press, San Diego, CA, 2001, 3-255.

[5] G. M. Schütz, J. Stat. Phys., 88:1-2 (1997), 427-445, arXiv: cond-mat/9701019.

[6] V.B. Priezzhev, Pramana, 64:6 (2005), 915-925.

[7] A. Rákos, G. M. Schütz, J. Stat. Phys., 118:3-4 (2005), 511-530, arXiv: cond-mat/0405464.

[8] A. M. Povolotsky, V.B. Priezzhev, J. Stat. Mech., 7 (2006), P07002, 28 pp., arXiv: cond-mat/0605150.

[9] V. B. Priezzhev, Phys. Rev. Lett., 91:5 (2003), 050601, 4 pp., arXiv: cond-mat/0211052.

[10] A. M. Povolotsky, V.B. Priezzhev, J. Stat. Mech., 8 (2007), P08018, 27 pp., arXiv: 0706.1890.

[11] K. Johansson, Commun. Math. Phys., 209:2 (2000), 437-476, arXiv: math/9903134.

[12] T. Nagao, T. Sasamoto, Nucl. Phys. B, 699:3 (2004), 487-502, arXiv: cond-mat/0405321.

[13] T. Sasamoto, J. Phys. A, 38:33 (2005), L549-L556, arXiv: cond-mat/0504417.

[14] A. Borodin, P. L. Ferrari, M. Prähofer, T. Sasamoto, J. Stat. Phys., 129:5-6 (2007), 1055-1080, arXiv: math-ph/0608056.

[15] A. Borodin, P. L. Ferrari, M. Prähofer, Int. Math. Res. Pap., 1 (2007), rpm002, 47 pp.

[16] A. Borodin, P. L. Ferrari, T. Sasamoto, Comm. Pure Appl. Math., 61:11 (2008), 1603-1629, arXiv: math-ph/0703023.

[17] T. Imamura, T. Sasamoto, J. Stat. Phys., 128:4 (2007), 799-846, arXiv: math-ph/0702009.

[18] A. Borodin, P. L. Ferrari, Electron. J. Probab., 13 (2008), 1380-1418.

[19] A. Borodin, P. L. Ferrari, T. Sasamoto, Commun. Math. Phys., 283:2 (2008), 417-449, arXiv: 0707.4207.

[20] M. Kardar, G. Parisi, Y.-C. Zhang, Phys. Rev. Lett., 56:9 (1986), 889-892.

[21] M. Prähofer, H. Spohn, Phys. Rev. Lett., 84:21 (2000), 4882-4885, arXiv: cond-mat/9912264.

[22] P. L. Ferrari, J. Stat. Mech., 7 (2008), P07022, 17 pp., arXiv: 0806.1350.

[23] A. M. Povolotsky, V.B. Priezzhev, G. M. Schütz, J. Stat. Phys., 142:4 (2011), 754-791, arXiv: 1007.1391.

[24] J. G. Brankov, V. B. Priezzhev, R. V. Shelest, Phys. Rev. E, 69:6 (2004), 066136.

[25] A. Borodin, E. M. Rains, J. Stat. Phys., 121:3-4 (2005), 291-317, arXiv: math-ph/0409059.

[26] H. Rost, Z. Wahrsch. Verw. Gebiete, 58:1 (1981), 41-53. 